\title{
ACREDITACIÓN DE LA CALIDAD Y SUS RETOS A LA GESTIÓN
}

\author{
Dr. Otoniel Alvarado Oyarce
}

\begin{abstract}
RESUMEN
En el presente artículo se presentan las inquietudes, desde una óptica estrictamente operativa, que tanto los resultados de la autoevaluación, la evaluación externa, el mejoramiento de la calidad, son producto de la gestión y que el mantenimiento de la misma una vez certificada (acreditada) la institución será consecuencia -también- de los mecanismos de gestión que se adopten.
\end{abstract}

Una de las posibles estrategias para enfrentar la crisis educativa, sobre todo en los países subdesarrollados, consiste en llevar adelante programas de evaluación (autoevaluación específicamente) para efectos de la consecuente acreditación de la calidad educativa. Es así como las universidades de nuestro país, con mayor énfasis que las entidades de otros niveles educativos, están viviendo la vorágine de la autoevaluación, para efectos de la acreditación respectiva, con la expectativa que logrados dichos procesos puedrm mejorar sus niveles de calidad $y$ asumir el reto de la competitividad, en el marco de la globalización. Como siempre en nuestro país, muy a la zaga de otros, tardíamente se ha comenzado con estos procesos cuando en otras latitudes ya lo vienen haciendo desde la década pasada, por lomenos.

Sin embargo, nunca es tarde para formular algunas reflexiones, desde otras dimensiones, que posibiliten un mayor entendimiento, mejor aplicación y resultados del proceso en ciernes, en este caso en torno a algunas ideas sobre los problemas en la gestión educativa que dicho proceso conlleva.

\section{ABSTRACT}

From an operative view, all concerns in this research about the results of the self-evaluation, the externa! evaluation, and the quality improvement are the result of management and maintaining this after being certified (accredited) the institution will also be the consequence ofthe management mechanisms to adopt.

One of the possible strategies to cope the educational crisis, in non developed countries, is implementing evaluation programs (specifically self-evaluation) to the consequent accreditation of the educational qua lit y. In this way, the universities of our country, in a more emphatic way than other educational institutions, are experimenting the self-evaluation, to the respective accreditation, waiting that those processes could improve their quality levels and be competitive in globa li zat ion. As usual in our country and very different from others, we have started very late with these processes when in other regions this has been done from the last decade.

However, it is not late to formulate sorne ideas, from other views to enable a great comprehension, best application and result of process in the making, in this case ideas about educational management problems resulting from the process.

\section{PALABRAS CLAVE}

Acreditación, autoevaluación, evaluación externa, certificación, gestión.

\section{KEYWORDS}

Acreditation, self-evaluation, externa! evaluation, certification, management. 


\section{Antecedentes}

A 1 margen de los problemas derivados de la crisis. universita ia, y educativa en general, que vienen acaeciendo en nuestro país desde hace un buen tiempo, los procesos mundiales de globalización acentúan aún más estos problemas, con el riesgo de quedar fuera del ranking mundial y, por ende, de la competencia internacional.

Precisamente, uno de los problemas causantes de la crisis educativa -desde nuestra perspectiva profesional- radica en la deficiente gestión que se viene ejerciendo en las distintas entidades e instancias educativas del país, por ser el área más sensible a las deficiencias e inmoralidades.

Otra de las razones que ocasiona esta crisis radica en la masificación de las instituciones educativas, sobre todo universitarias, en razón a la liberalización del servicio educativo, enla mayoría de los casos con una fuerte tendencia hacia la mercantilización y el rentismo aultranza.

Frente a estas consideraciones se ha planteado entonces, como una estrategia de solución, el desarrollo de procesos de autoevaluación y acreditación, mediante el sistema denominado Sistema Nacional de Evaluación y Acreditación de la Calidad Educativa (SINEACE), iniciándose a nivel universitario enlasáreas de educación ydemedicina, respectivamente. Sinembargo, como estos procesos son autónomos y con marcados énfasis en aspectos básicamente formales, nos queda la duda -ojalá infundada- que se pueda ejecutar solo con fines burocráticos.

\section{El proceso}

\section{Primer paso: El proceso de autoevaluación}

Partimos de la premisa que la autoevaluación mide los resultados de la gestión en un momento determinado (transversal); por lo tanto, cualquiera que fueren los resultados a que se arriben, los mismos recaerán necesariamente en la calidad de la gestión aplicada hasta ese momento. Es decir, un fenómeno de causa efecto o por lo menos de correlación significativa irrebatible .

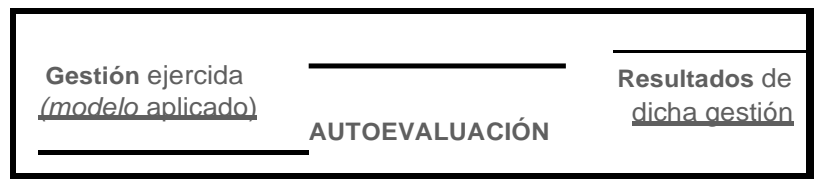

Parael casodelasentidades estatales, por elhechode estar íntimz.:nente ligado al entramado jurídicoadministrativo del estado, fácilmente devienen o recaen en enfoques burocráticos en la gestión, enfoque que como se sabe, si bien es necesario o útil, un fácil y excesivo apego al mismo resulta pernicioso para eldesarrollo institucional.

En el caso de la gestión universitaria estatal en nuestro país, de acuerdo a su tradición y normatividad, tiene la característica de ser intermitente en cuanto que las autoridades (de gobierno) son elegidas periódica y alternativamente, en procesos en los cuales las luchas por el poder salen a flote $y$, como consecuencia de las elecciones, se generan divisiones en grupos muchas veces irreconciliables y que hacen difícil una gestión participativa y exitosa. Asimismo, como las autoridades resultan de procesos eleccionarios, en muchos casos no siemprese eligen alos másidóneos para la gerencia de sus respectivas unidades académicas, o en otros casos, rápidamente, pierden sulegitimidad.

Es por esta razón, entre otras, en nuestro modesto entender, que los conceptos y técnicas modernas de gestión como el desarrollo organizacional, el coaching, la cultura y el clima institucional, la gestión estratégica y otras, no tienen cabida en el ámbito universitario, por tanto nos quedamos en enfoques clásicos como el burocrático y el conductista, por ser quizás los más llevaderos, por ser fáciles de aplicar.

Posiblemente, existan otras causas más que ocasionen o agraven aún más esta situación, como por ejemplo, la carencia de recursos, la masificación universitaria, la incoherencia o carencia de políticas universitarias, etc., sobre las cuales cada universidad tiene muy poco que hacer y mucho que soportar o acondicionarse.

En conclusión, los reportes de la autoevaluación, responden necesariamente a las calidades de la gestión aplicadas hasta ese momento .

\section{Segundo paso: Propuestas de mejora}

Como resultados -previsiblemente deficitarios- de la autoevaluación, el siguiente paso constituye la propuesta y la aplicación de diversas medidas de mejora en los distintos aspectos evaluados. Es aquí donde se hace necesario, nuevamente, el "enrumbamiento" de las acciones de gestión, las mismas que estarán supeditadas a las capacidades gerenciales y de

\section{1 un1Fé}


decisión de las autoridades respectivas, cuando no a las condiciones organizacionales vigentes .

Posiblemente será necesario adoptar nuevas estrategias de gestión, por ejemplo concertación entre grupos antagónicos u opositores, alianzas y convenios con otras entidades que aporten al desarrollo ins $\mathrm{t}$ i $\mathrm{t} \mathrm{u}$ ci on al ( el Consorcio de Universidades Estatales es un buen ejemplo), capacitación de los agentes intervm1entes, programas de sensibilización del personal involucrado, máxime si no están comprometidos o involucrados en el proceso.

Se busca entonces un adecuado equilibrio en la siguiente secuencia lógica:

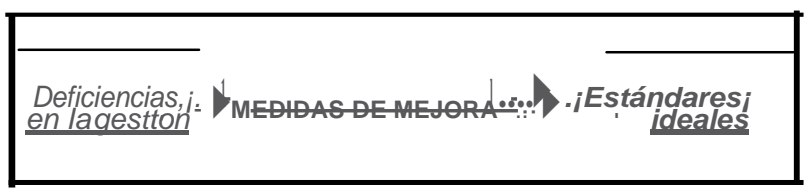

Como paso definitivo en el proceso, luego de lograr los resultados ideales deseables, viene la validación externa, que puede ser definitiva o no, dependiendo de los resultados a que arribe el agente acreditador. En el caso de que hubiera correcciones que efectuar, nuevamente la intervención de los gestores o decisores respectivos se hará indispensable hasta llegar a los niveles requeridos para la aprobación definitiva. Es de esperar que los procesos de transferencias y rotaciones en el poder no dificulten la consecución de los estándares ideales con los cuales se debelograr la tan ansiada acre ditación.

\section{Tercer paso: Mantenimiento de la calidad}

lograda la acreditación anhelada, obviamente corresponde mantener el nivel de calidad alcanzado, tratando en lo posible de ir superándolo cada vez, lo que a su vez requiere de nuevos patrones culturales para internalizar el compro-miso y esfuerzo de los grupos que acceden al gobierno universitario.

En relación a este rubro, debemos indicar que el mantenimiento o aseguramiento de la calidad supone dos niveles o tipos de requerimientos específicos:

AcreditaciónI IMantenimientol superación de estándares
El primer nivel está relacionado con el cumplimiento del estándar 8 de la 10 dimensión del modelo formulado por CONEAU, en el cual se plantea la necesidad de que cada entidad deba implementar un sistema degestión dela calidad.

En este contexto, entendemos por sistema de gestión de calidad el (o los) programa(s) informático(s) pertinente(s) que permita(n) controlar y monitorear el cumplimiento de cada uno de los estándares de calidadlogrados, a efecto dequenodecaiganenalgún momento, máxime si la acreditación tiene como característica la de ser periódica. En otras palabras, se requiere de implementar sistemas de información gerencia! (SIG) específicos que ayuden a tomar las decisiones académicas y administrativas pertinentes. El segundo tipo de requerimiento está directamente relacionado con el primero, en tanto que la aplicación de los sistemas informáticos, en tanto herramientas, requieren de las habilidades sociales (actitud gerencial) de los decisores a efectos de que puedan lograr la aceptación y cumplimiento de las medidas adoptadas, dentro de las cuales seguramentenohandefaltarmuchas innovaciones, y que como tales en muchos casos resulta difícil introducirlas, dada la resistencia de sus ejecutores, sobre todo si el clima y la cultura organizacional no son favorables .

Pero no solamente serán necesarias las habilidades sociales, también se requieren muchas habilidades o competencias cognitivas para aplicar nuevas técnicas o modelos de gestión modernos, dentro de las cuales la gestión estratégica, la gestión del conocimiento, el BSC, el EFQM, etc., y tantas otras que reporta la teoría administrativa vigente .

\section{En síntesis:}

lQ la autoevaluación y la acreditación en última instancia miden los resultados de la gestión.

2Q El mantenimiento o aseguramiento de los niveles de calidad, alcanzados con la acreditación, requieren necesariamente de enfoques, técnicas e instrumentos de gestión que haga posible dicho cometido, para cuyo efecto sería recomendable que, en lo sucesivo, quienes aspiren a cargos directivos lleguen premunidos del conocimiento y praxis de la tecnología administrativapertinente. 


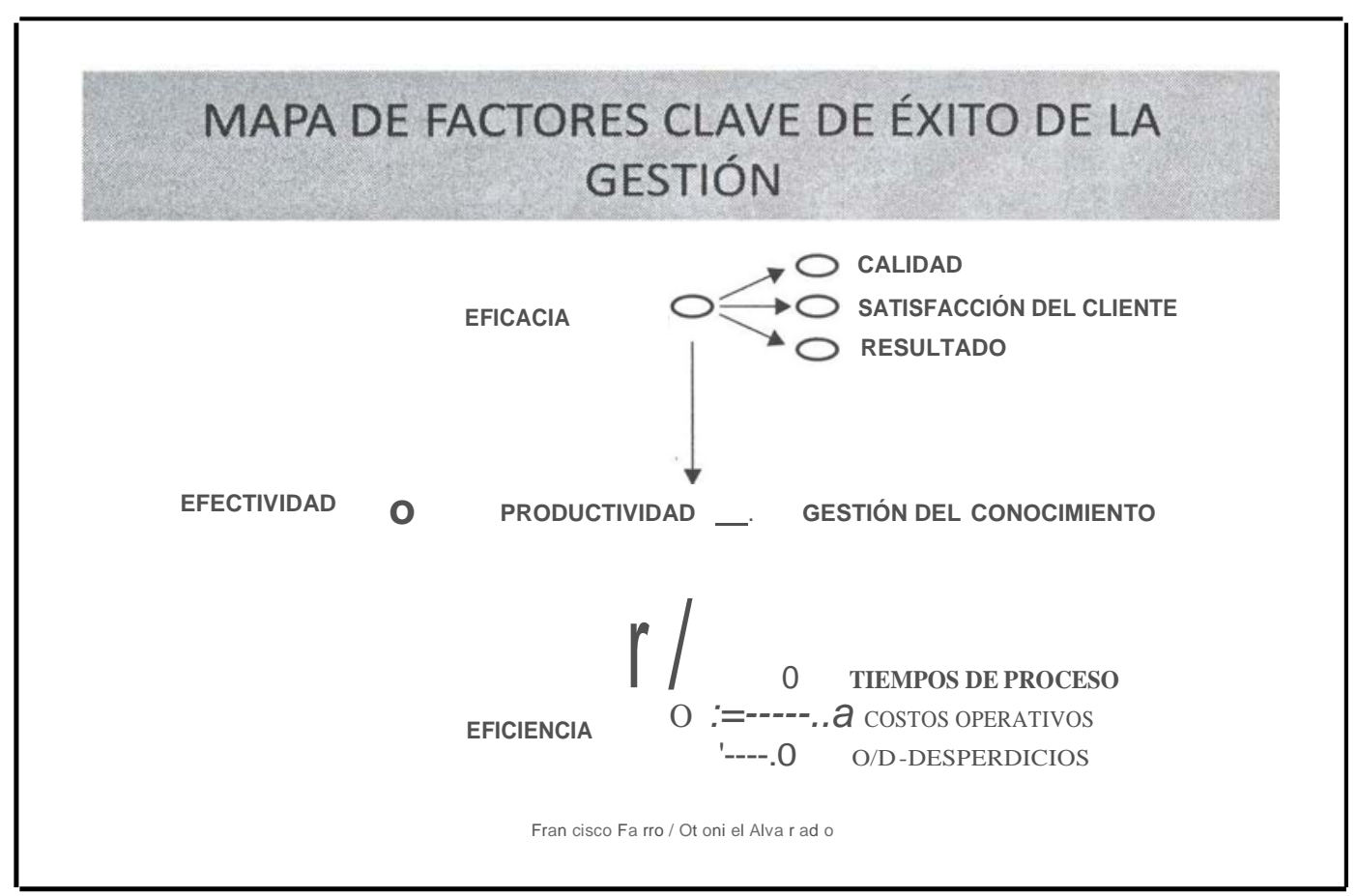

Dr. Otoniel Alvarado Oyarce

Profesor y Licenciado en Administración y Magíster en Administración de la Educaci ón. Ha sido funcionario del Ministerio de Educación en cargos técnicos y direct ivos. Es profesor de diversas Escuelas de Posgrado. Ha escrito un buen número de libros de su especialidad. Ha sido premiado con las Palmas Magisteriales en los Grados de Maestro y Amauta, respectivamente.

\section{1 un1Pé}




\section{MAPA DE FACTORES CLAVE DE ÉXITO DE LA GESTIÓN}

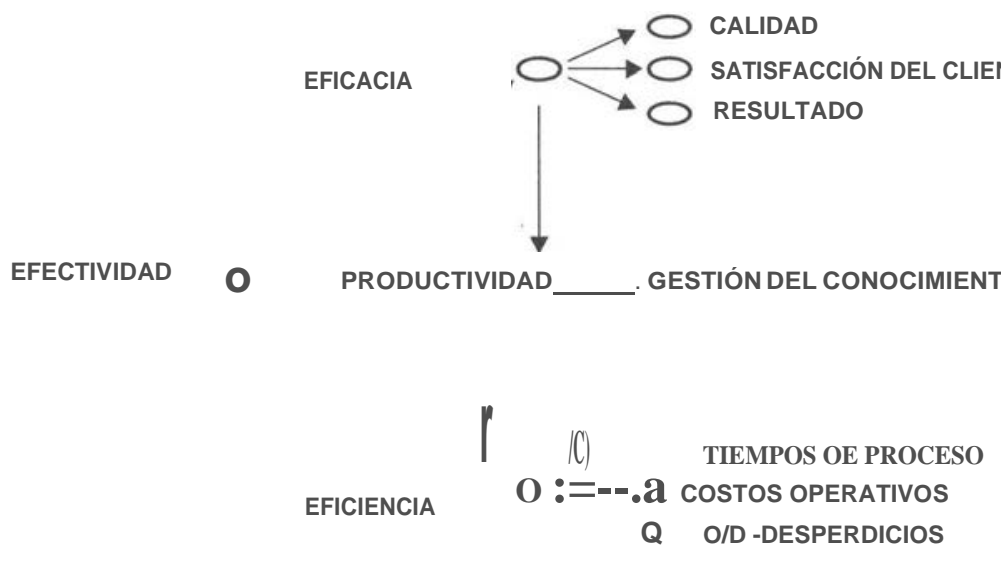

Dr. Otoniel Alvarado Oyarce

Profesor y Licenciado en Administración y Magíster en Administración de la Educación. Ha sido funcionario del Ministerio de Educación en cargos técnicos y directivos. Es profesor de diversas Escuelas de Posgrado . Ha escrito un buen número de libros de su especialidad. Ha sido premiado con las Palmas Magisteriales en los Grados de Maestro y Amauta, respectivamente. 